\title{
Application of Multi-Pixel Photon Counter to Positron Emission Tomography
}

\author{
Martin Göttlich, Erika Garutti, Valentin Kozlov, Hans-Christian Schultz-Coulon, Alexander Tadday, Adel Terkulov
}

\begin{abstract}
The Multi-Pixel Photon Counter (MPPC) is a silicon based photon counting device consisting of multiple Avalanche Photodiodes operated in Geiger mode. In order to detect $511 \mathrm{keV}$ photons we use MPPC devices with an active area of $3 \times 3 \mathbf{m m}^{2}$ which are directly coupled to $3 \times 3 \times 15 \mathbf{m m}^{3}$ scintillator crystals made of Lutetium Fine Silicate (LFS). Two gammadetectors of this kind are used to detect both coincident photons emerging from an $e^{+} e^{-}$annihilation. The energy and the time resolution are measured. The very good performance of the gamma-detectors motivate the next step towards building a gamma camera consisting of a matrix of MPPC devices and scintillator crystals. First results concerning the design and the homogeneity of the read-out channels are presented. About 100 LFS crystals are tested. First studies on the properties of the ASIC which will be used for the multi-channel read-out are discussed. The experimental studies are supplemented with Monte Carlo simulations based on GEANT4. A full PET system is simulated in order to study and optimize the performance of the system in terms of spatial resolution.
\end{abstract}

Index Terms-MPPC, PET.

\section{INTRODUCTION}

$\mathbf{P}$ OSITRON Emission Tomography (PET) is a non-invasive medical imaging technique. A $\beta^{+}$emitter is used to mark a tracer which is injected into a living organism. The two $511 \mathrm{keV}$ photons, produced by the $e^{+} e^{-}$annihilation inside the organism, are detected in coincidence and their line of response is identified. The reconstruction of several lines of response allows to retrieve a functional image of the tissue in which the tracer is absorbed. The energy and the time resolution of the used gamma-detectors are two important parameters which determine the overall performance of the PET scanner. A good energy resolution is crucial in order to reject photons which have undergone Compton scattering inside the organism and thus changed their direction. The time resolution of the gamma-detectors determines the minimal width of the coincidence window and thus the amount of background arising from random coincidences. Furthermore, the time information can be used to directly improve the spatial resolution as done in the time-of-flight PET. Apart from the requirements on the energy and time resolution a small pixel size of the PET camera is required since this mainly determines the spatial resolution. In the present work it will

This work is supported by the Helmholtz-Nachwuchsgruppen grant VHNG-206 and by the DFG research and training group IRTG 1039/1.

Erika Garutti and Martin Göttlich are with the DESY research institute, Notkestraße 85, D-22606 Hamburg, Germany.

Hans-Christian Schultz-Coulon and Alexander Tadday are with the University of Heidelberg, Im Neuenheimer Feld 227, D-69120 Heidelberg, Germany

Valentin Kozlov and Adel Terkulov are with the Lebedev Physical Institute, Moscow

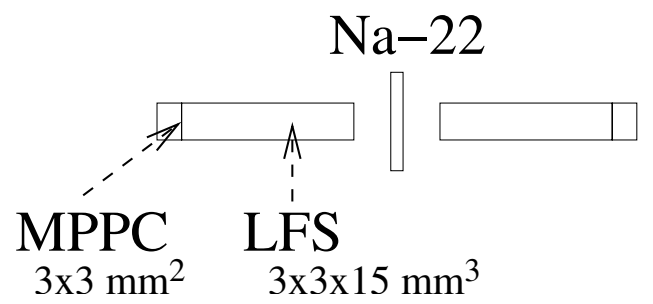

Fig. 1: Experimental setup.

be demonstrated that a gamma-detector consisting of a fast scintillator crystal (Lutetium Fine Silicate [2]) read out by a Multi-Pixel Photon Counter from Hamamatsu well meets the mentioned requirements. The MPPC is a very compact device with excellent photon counting capability which can be operated at low voltage and which is insensitive to magnetic fields. It shows a high sensitivity in the $420 \mathrm{~nm}$ spectral region. For more details see [1]. This matches the emission spectra of fast crystal scintillators which are peaked in the blue and ultra-violet spectral region, yielding a high photondetection efficiency. The outline of this article is as follows. The basic properties in terms of energy and time resolution of a single gamma-detector consisting of an MPPC and a scintillator crystal are presented in section II. In section III we present our work towards a first PET scanner prototype. Monte Carlo simulation studies based on GEANT4 are performed to investigate the anticipated spatial resolution of the system. At the end of this article we give a conclusion and an outlook.

\section{ENERGY AND TIME RESOLUTION}

The experimental setup used to determine the energy and time resolution of a system of two gamma-detectors is shown in figure 1. An individual gamma-detector consists of a scintillator crystal (LFS, $3 \times 3 \times 15 \mathrm{~mm}^{3}$, developed by General Physics Institute Moscow) wrapped in Teflon and an MPPC device from Hamamatsu with a sensitive area of $3 \times 3 \mathrm{~mm}^{2}$ and 3600 pixels. As $\beta^{+}$emitter a Na-22 source is used. The signals from the MPPCs are digitized without any amplification. The charge integration is performed by a VME QDC CAEN V965A module, gated by a coincidence of two MPPC signals generated using standard NIM logic. For the time measurement a VME TDC CEAN V488N module is used. The 'start' and 'stop' signals are generated by a low threshold discriminator NIM module. Each coincidence defines an event for which the measured energies and the time difference between the signals are recorded. The energy resolution amounts to $11 \%$ FWHM. For more details concerning the energy resolution 


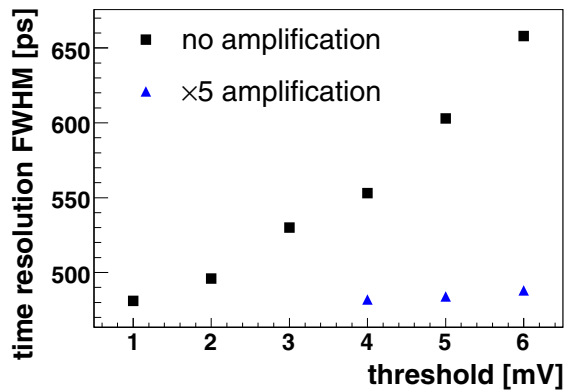

Fig. 2: Time resolution as a function of the discriminator threshold.

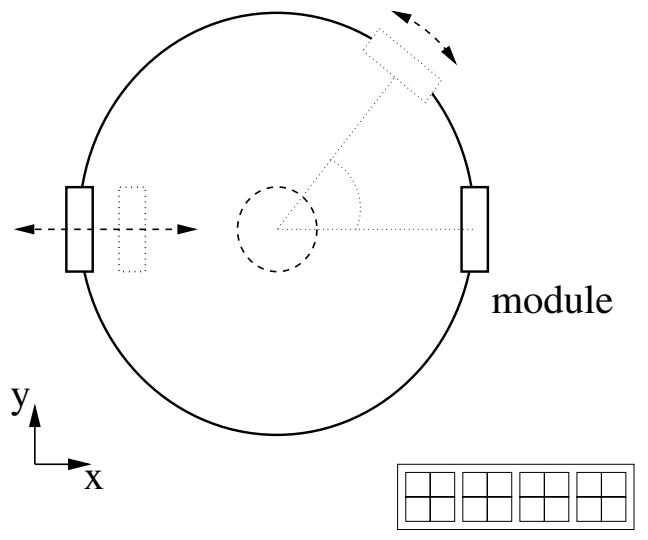

Fig. 3: Sketch of the PET scanner prototype.

we refer to a previous publication [3]. Figure 2 shows the time resolution as a function of the discriminator threshold. Only photo-eletric events are considered. The time resolution gets worse with higher thresholds due to larger time walk. The usage of signal amplifiers does not improve the time resolution. We plan to perform measurements with Amplitude and Rise-time Compensated triggering since time jitter due to noise is limiting the precision.

\section{TOWARDS A FIRST PROTOTYPE}

The next step after investigating the performance of single crystals read out by MPPC devices is the construction of a small size prototype. The purposes of this prototype are manifold. On one side the channel-to-channel homogeneity and reproducibility of the concept has to be tested. A solution for the necessary multi-channel readout has to be found which is scalable to a larger prototype for commercial use. The calibration and monitoring requirements of a multi-channel detector need to be addressed as well as the stability of operation. On the other hand, a small prototype will give the opportunity to test the improvement of time resolution in the 2D and maybe 3D spatial reconstruction of a non-point-like radioactive source.

The layout of the prototype is sketched in figure 3 . The scanner consists of 2 modules. In order to detect all possible lines of response the modules are rotated around the centre of the field of view by means of a high precision rotation stage. In addition each module can be individually moved in radial

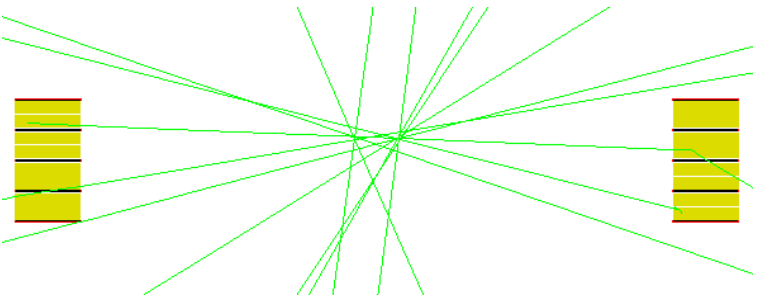

Fig. 4: View of the PET scanner.

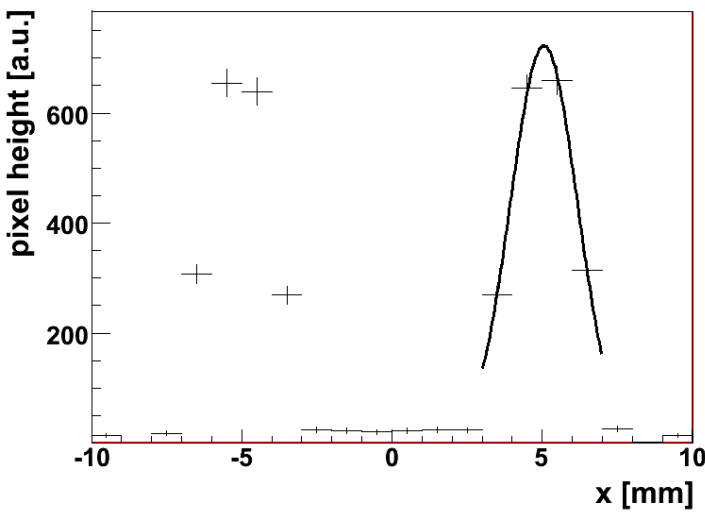

Fig. 5: Profile of the reconstructed image along the $\mathrm{x}$ axis.

direction and the angle between the modules can be changed. Each module consists of four submodules. A submodule is a $2 \times 2$ matrix of $3 \times 3 \times 15 \mathrm{~mm}^{3}$ crystals which are read out by a $2 \times 2$ MPPC array from Hamamatsu. Each MPPC is read out individually, for a total number of 16 channels per module. It is forseen to build two additional modules to reduce the scan time which will give 64 read-out channels in total. In the following studies towards building a first prototype are presented. Monte Carlo simulation studies concerning the performance of the prototype, the multi-channel read out using ASIC technology, the crystal homogeneity and the properties of the submodules in terms of energy resolution are addressed.

\section{A. Monte Carlo simulation studies}

In order to study the performance of the prototype in terms of spatial resolution, Monte Carlo simulation studies are performed using the GATE toolkit [4] which is based on GEANT4. Figure 4 shows the detector geometry as described previously. The energy and time resolution of each single detector consisting of a $3 \times 3 \times 15 \mathrm{~mm}^{3}$ LFS crystal read out by an MPPC is tuned to match the laboratory measurements presented above. For very basic studies the response of the scanner to two point-like radioactive sources at $(-5,5) \mathrm{mm}$ and $(5,5) \mathrm{mm}$ is simulated. Images are reconstructed from the raw data according to the following procedure:

1) An energy cut is applied to select photo-electric events.

2) Coincidences are formed from events in different modules if the time difference between the measurements is smaller than 2 ns.

3) Each coincidence gives a line of response defined by the two crystal centres. 
4) All information are collected in a sinogram.

5) An image is reconstructed from the sinogram using the Filtered Backprojection Algorithm (FBP) applying a ramp filter.

Figure 5 shows the profile of the reconstructed image along the $\mathrm{x}$-axis through the two sources. The two source are well separated. According to Gaussian fit the spatial resolution amounts to $2.6 \mathrm{~mm}$ FWHM.

\section{B. Multi-channel read out}

For the multi-channel read out we want to make use of an adapted version of the SiPM Integrated Read-Out Chip (SPIROC) [5] which is a very front-end electronics for an ILC prototype hadronic calorimeter with Silicon photomultiplier readout. This ASIC is due to equip a 10,000-channel demonstrator in 2009. It has been realized in $0.35 \mu \mathrm{m} \mathrm{SiGe}$ technology. The SPIROC has been developed to match the requirements of a large dynamic range, low noise, low power consumption, high precision and large number of readout channels needed. It is an auto-triggered, bi-gain, 36-channel ASIC which allows to measure on each channel the charge from one photoelectron to 2000 and the time with a 100 ps accurate TDC. An analogue memory array with a depth of 16 for each channel is used to store the time information and the charge measurement. A 12-bit Wilkinson ADC has been embedded to digitize the analogue memory content (time and charge on 2 gains). The data are then stored in a 4 kbytes RAM. A digital part has been integrated to manage all these features and to transfer the data to the data acquisition. Each scanner module will be read out by an individual SPIROC and coincidence will only be formed between different modules, i.e. the time jitter between measurements from different SPIROC chips is an important quantity to measure.

Figure 6 shows a test-board which is equipped with a SPIROC ASIC and an ALTERA FPGA for configuring the chip. The slow control is done via USB. In a first step we studied the properties of the SPIROC for the read-out of MPPC devices which are illuminated by light from an LED. The time jitter between the two triggers delivered by two test-boards which are connected to an MPPC device, is measured to be 100 ps FWHM. In a next step we measured the time jitter between two coincident signals from a Na-22 source with the setup shown in figure 1 . For the time resolution we obtained about 5 ns FWHM. This measurement suffered from the fact that one board was not working properly, i.e. it was not possible to set the threshold to a reasonable low value. We are confident that with new versions of the test-boards we reach values well below $1 \mathrm{~ns}$.

The SPIROC was not developed for continuous operation but for the ILC beam structure. It has to be studied if this imposes a problem and we have to solve the issue of synchronizing the different SPIROC chips reading out the scanner modules.

\section{Crystal homogeneity}

In order to investigate the homogeneity of the LFS crystals we measured the energy and time resolution with the experimental setup depicted in figure 1 where we kept one channel

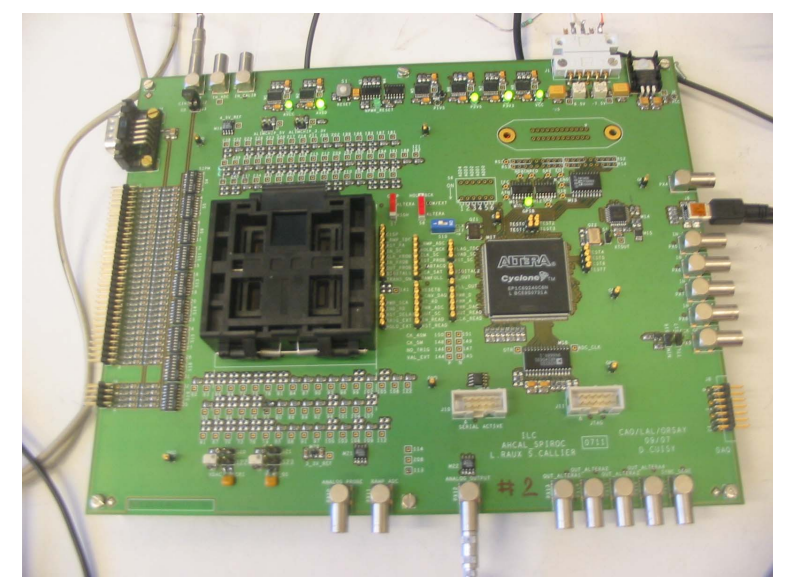

Fig. 6: The test-board with the SPIROC and the ALTERA FPGA to configure the ASIC.

unchanged while the other channel was used to test 100 crystals. The energy mean resolution is $12 \%$ with a standard deviation of $0.6 \%$. For the time resolution we obtained a mean of 460 ps with a standard deviation of 13 ps. Within the experimental precision the crystals are homogeneous in terms of energy and time resolution. The geometrical tolerances are $200 \mu \mathrm{m}$ which has to be taken into account in the design of the submodules, i.e. the $2 \times 2$ matrices of crystals and MPPC devices.

\section{Crystal matrix}

First tests were performed to study the properties of a $2 \times 2$ crystal matrix read out by a $2 \times 2$ array of MPPC devices. The MPPC array developed by Hamamatsu which we use for the present study consists of four MPPC devices with a sensitive area of $3 \times 3 \mathrm{~mm}^{2}$ and 3600 pixels each. The energy resolution at $511 \mathrm{keV}$ of each single channel is determined. Different configurations are tested: a crystal matrix out of bare crystals, a matrix of crystals which are laminated with reflective foil. The later configuration is tested with and without optical grease. Figure 7 shows the energy resolutions for each individual MPPC device in the $2 \times 2$ array. The best result is obtained for crystals wrapped in reflective foil which are optically coupled to the MPPC devices using grease with an reflective index similar to that of the crystals. The measured energy resolutions are worse than the results obtained previously for single crystals. In addition the response of the four channels is rather different. One reason for this is that the same bias voltage is applied to the individual MPPC devices allthough they have a different break-down voltage. This was done to keep the read-out electronics as simple as possible for the very first measurements; this will be changed in future.

Another reason is imperfect alignment of the individual crystals with respect to the MPPC devices. Both problems will be addressed in the design of the submodules.

\section{CONCLUSION AND OUTLOOK}

It has been demonstrated that MPPC devices are very suitable for the read-out of crystal scintillators. The good 


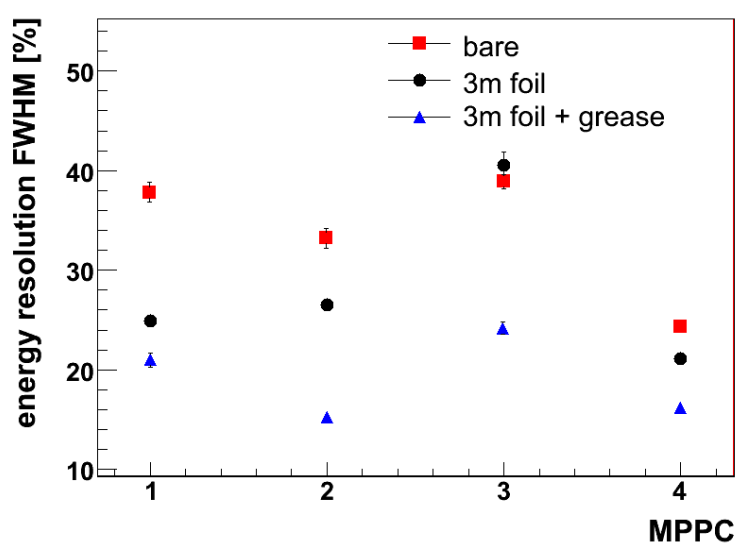

Fig. 7: The energy resolution for individual MPPC devices in a $2 \times 2$ matrix for different configurations.

energy resolution (12\% FWHM) allows to distinguish between Compton and photo-electric events allowing to reject scattered events very efficiently as is necessary for the PET application. The time resolution of 460 ps FWHM obtained with leading edge triggering allows to choose a small coincidence time window reducing the number of random coincidences and to use time-of-flight information to considerably improve the signal to background ratio of the reconstructed images as discussed in [6].

Towards a first prototype several key studies were performed. According to Monte Carlo simulations based on GEANT4 we expect a spatial resolution of $2.6 \mathrm{~mm}$ FWHM which is a factor two better than what is achieved with present clinical PET scanners. The sensitivity has still to be studied. Concerning the multi-channel read-out we demonstrated that the SPIROC technology gives very promosing results, i.e. the timing performance is suitable for the projected prototype. Still the complete read-out scheme has to be developed. In addition the channel homogeneity was found to be sufficient for the PET application.

In future we plan to test the newly developed $4 \times 4$ MPPC arrays from Hamamatsu. We also want to investigate other scintillator materials, i.e. $\mathrm{LaBr}_{3}: \mathrm{Ce}$ which provides better energy and time resolution.

\section{ACKNOWLEDGMENT}

We thank Hamamatsu, which kindly provided us the tested samples of MPPC. A further thank to P. Smirnov and U. Cornett for their support in establishing the laboratory setup.

\section{REFERENCES}

[1] Hamamatsu, Multi-Pixel Photon Counter, Datasheet.

[2] A. I. Zagumennyi, Yu. D. Zavartsev, S. A. Kutovoi, Patent US 7,132,060 PCT Filed:Mar.12, 2004.

[3] N. D'Ascenzo, E. Garutti, M. Goettlich, H. C. Schultz-Coulon and A. Tadday, "Study of Micro Pixel Photon Counter for the Application to Positron Emission Tomography," arXiv:0805.0525 [physics.med-ph].

[4] S. Jan et al., GATE : a simulation toolkit for PET and SPECT, Phys. Med. Biol. 49, 4543 (2004) [arXiv:physics/0408109].

[5] M. Bouchel, F. Dulucq, J. Fleury C. de La Taille, G. Martin-Chassard, L. Raux, SPIROC (SiPM Integrated Read-Out Chip): Dedicated very front-end electronics for an ILC prototype hadronic calorimeter with SiPM read-out Nuclear Science Symposium Conference Record, 2007.
[6] W. W. Moses and S. E. Derenzo, Prospects for time-of-flight PET using LSO scintillator, IEEE Trans. Nucl. Sci., NS-46, 474-478, (1999). 